\title{
Viola Heutger*
}

\section{Der Platz der juristischen Fachsprache in der Experten-Laien-Kommunikation}

\begin{abstract}
In this commentary, the author outlines possible contributions of a linguistic monitoring especially to the interpretive work of legal experts from the point of view of a lawyer. However, for such a monitoring to be efficient more efforts must be made from the part of the linguists to make clear what the specific methodological characteristics of a linguistic monitoring consist in. The article sees possible contribution in fields like interpreting legal texts in more languages like in the EU system, overcoming the differences of legal systems in international legal cooperation, and optimizing national legal texts produced on the basis of community legal texts in order to make them more accessible for national citizens.
\end{abstract}

\section{1. $\quad$ Einleitung}

Als Jurist wird man regelmäßig in Gesprächen mit Nichtjuristen konfrontiert mit der Aussage, dass die deutsche Rechts-, Gesetzes- und Verwaltungssprache schwerverständlich sei. Das Juristendeutsch wird als kompliziert, langatmig und trocken definiert. Da der Jurist Lösungen finden muss für Probleme des Alltags, stellt sich mit Recht die Frage, ob die juristische Fachsprache nicht dichter bei den Bürgern stehen kann.

Der Umgang mit Texten ist jedem Juristen vertraut, die Auslegung seines Inhalts kann jedoch Schwierigkeiten für Nichtjuristen in sich bergen. Mit einem angelernten Fachwissen begegnet der Jurist Texten und legt diese mit Hilfe eines spezifischen Interpretationsinstrumentariums aus. In der Regel wendet der Jurist dabei die folgenden Interpretationsmethoden an:

\footnotetext{
* Dr. Viola Heutger

Vrije Universiteit Amsterdam

v.heutger@rechten.vu.nl
}

Hermes - Journal of Language and Communication Studies no 36-2006 
1. die grammatikalische Interpretation, die auch Wortlaut-, Wortsinnoder Verbalinterpretation genannt wird ${ }^{1}$

2. die systematische Interpretation, die nach dem Regelungszusammenhang fragt,

3. die historische Interpretation, die nach der Entstehungsgeschichte und der Regelungsabsicht des Gesetzgebers forscht

4. sowie die objektiv-teleologische Interpretation ${ }^{2}$.

Dem Juristen steht somit ein anderes, wenn auch zum kleinen Teil ähnliches Textauslegungsinstrumentarium zur Verfügung als dem Linguisten.

Da der Jurist sich in der Regel in seinem Umgang mit Texten sehr festgelegt fühlt, wäre ein interdisziplinärer Ansatz sicher ein guter Weg in die Richtung eines neuen Umgangs mit juristischen Texten. Der Blick eines Nichtjuristen auf die Texte eines Juristen und eine daran anschließende Analyse wird jedem Juristen neue Einsichten bieten können, wie juristische Texte beim nichtjuristischen Empfänger ankommen.

Auf der Tagung der Gesellschaft für angewandte Linguistik (GAL) in Koblenz im September 2005 wurde ein Themenbereich dem Thema: „Linguistisches Monitoring der juristischen Experten-Laien-Kommunikation“ gewidmet. Als sprachlich interessierte Juristin durfte ich daran teilnehmen und möchte hier nun gerne einige Eindrücke und Stellungnahmen zu diesem Thema aus juristischer Sicht äußern.

\section{Linguistisches Monitoring}

Das linguistische Monitoring beschäftigt sich mit einer strukturierten und langfristigen Beobachtung von bestimmten Diskursgruppen, wie z.B. Verwaltungsbeamte mit Antragstellern oder Anwälte mit Klienten. Die derzeit untersuchten und in Koblenz auf der GAL-Tagung vor-

\footnotetext{
1 Hierbei wird die Bedeutung des Normtextes aus den darin verwendeten sprachlichen Ausdrücken ermittelt. Auch gibt es die Möglichkeit, die Sprache aus der Zeit der Entstehung des Textes, die Sprache von heute oder die besondere Sprache von europäischen Dokumenten zu berücksichtigen.

2 „Teleologisch“ bedeutet zweckhaft, durch den Zweck bestimmt, es wird also nach Sinn und Zweck einer Regelung gesucht.
} 
gestellten Monitoring-Ergebnisse bezogen sich immer auf Gruppen, die durch eine Experten-Laien-Kommunikation gekennzeichnet waren. Da, wie oben bereits dargestellt, ein Jurist sich Texten auf eine ganz spezifische Art nähert, ist ein interdisziplinäres Nachdenken über eine Optimierung der Experten-Laien-Kommunikation sicher erkenntnisreich. Die Einführung eines linguistischen Monitorings und die allgemeine Zugänglichmachung der Ergebnisse erscheint mir daher sehr sinnvoll, auch wenn sie verschiedene noch offene Fragen aufwirft.

\subsection{Interdisziplinärer Ansatz des Monitorings}

Was ist nun genau linguistisches Monitoring aus der Sicht der angewendeten Methode? Genau bei dieser Frage liegt sicher die erste Schwierigkeit. Die in Koblenz vorgestellten Monitoring-Projekte ${ }^{3}$ waren aus meiner Sicht nicht rein linguistisch, sondern enthielten auch soziologische und verhaltenspsychologische Elemente. Wie bei jeder Analyse von menschlichen Interaktionen ist es schwierig, hier fachspezifisch vorzugehen. Es erscheint mir daher problematisch festzustellen, was am linguistischen Monitoring nun genau linguistisch ist. Wo begibt man sich in interdisziplinäre Bereiche oder ganz und gar in einen anderen Fachbereich? Methodisch gesehen wäre es interessant zu wissen, was nun genau die linguistische Methode wäre und welchen Erkenntnisgewinn sie ,rechtslinguistischen’ Vorgängen zufügen könnte.

Deutliche Standards und Methoden, die spezifisch sein sollten für ein linguistisches Monitoring, würden sicher zu einer besseren Verwendung der Ergebnisse eines Monitoring-Prozesses beitragen. Es wäre aber auch zu überlegen, ob ein Monitoring - gesehen von Aufwand, Zeit und Kosten - nicht von Anfang an interdisziplinär ausgerichtet sein sollte und sowohl juristische, soziologische, psychologische als auch linguistische Sachverhalte observieren, auswerten und beurteilen sollte. Fraglich bleibt in allen Fällen, wie man für die Zukunft Standards entwickeln könnte, die die Ergebnisse des Monitorings vergleichbar machen.

3 Siehe Einleitung zu diesen Themensektion. 


\subsection{Zusammenarbeit statt Kontrolle}

Ein Nachteil eines jeden Monitorings wird sein, dass zumeist ein Fremder in den ohnehin schon komplizierten Experten-Laien Diskurs hinzukommt. Dieses Eindringen sollte nicht unterschätzt werden, und es ist nicht auszuschließen, dass es bei der Untersuchung von mündlicher Kommunikation sogar einen Einfluss auf den Diskurs hat. Die normale Kommunikationsbeziehung sollte also so wenig wie möglich durch einen Monitoring-Mitarbeiter verstört werden. Das legt die Frage nahe, ob nicht in vielen Fällen eine Partei innerhalb der Experten-LaienKommunikation selber auch das Monitoring durch Ausfüllen von Fragebögen unterstützen könnte und somit ein direkter Dritter zur Aufzeichnung vermieden wird. Ein Monitoring durch eine Gruppe von Wissenschaftlern, die von außen in eine Institution für eine kurze Zeit hereinschaut, sollte vermieden werden. Der Prozess des Monitorings sollte auf jeden Fall begleitet werden von Menschen, die der Institution vertraut sind und die auch Lobbyträger für das Monitoring sind. Eine Interaktion erscheint mir zum Erfolg wichtig.

\subsection{Rechtsgewinn versus Erkenntnisgewinn}

Als Juristin frage ich mich, wo mir in der Zukunft ein linguistisches Monitoring helfen könnte. Der Umgang mit Texten ist ein großer Teil der alltäglichen Arbeit eines Juristen. Ständig ist man mit Situationen beschäftigt, die zum Nachdenken über im Text gebrauchte Begriffe auffordern. Die Auslegung von Begriffen ist nämlich eine der vornehmlichsten Aufgaben eines Juristen. Wie lege ich zum Beispiel den Begriff: unverzüglich aus? Im Studium wird gelehrt, dass das mit der Umschreibung „ohne schuldhaftes Zögern“ auszulegen sei. Sieht man nun aber eine Übersetzung, so steht da vielleicht im Englischen „,within in a reasonable time“. Wenn man nun zwei Sprachen nebeneinander legt, so ist es die Frage, ob dann noch die deutsche Formel des „ohne schuldhaftes Zögern“ angewendet werden sollte oder ob man versuchen sollte, einen gemeinsamen Standard für beide Sprachen zu finden. Sollte man also hier besser von der Person ausgehen, die unverzüglich handeln sollte? Oder von den Umständen, die den Fall umgeben? Wäre eine Antwort mit der Post bei einem electronic bill of lading, der Bananen auf hoher See zum Inhalt hat noch zeitgemäß? Oder muss immer der schnellste Weg genommen werden, also email oder Telefon? Wie soll man hier argumentieren? Helfen linguistische Methoden in so einem Fall? Für 
den Juristen stellt sich also die Frage, inwieweit ein linguistischer Textumgang dem Juristen bei der Auslegung von Rechtsproblemen helfen kann. Da Linguisten sich auch mit der Entwicklung der Sprache beschäftigen, könnte eine linguistische Auslegung hier helfen, sprachliche Gebräuche schneller zu identifizieren. Der Jurist ist es gewohnt Regeln als Korrektiv von Missständen in der Vergangenheit zu entwerfen und das Jetzt zu beobachten. Gesetzestexte sind daher immer eine Reaktion auf die Vergangenheit und sind zumeist zur Zeit ihres Inkrafttretens nicht mehr auf dem allerneusten Stand. Gerade in Bereichen des Transportrechts, des Telekommunikationsrechts und des Vertragsrechts sind Rechtsentwicklungen sehr schnell und oft nicht transparent. Neue Begriffe und Konzepte werden in diesen Bereichen zum Teil überschnell eingeführt. Denken wir nur an Begriffe wie: Handy, Franchising, Leasing, Timesharing, Fernabsatz, verbundene Rechtsgeschäfte und Verbraucherschutzmechanismen ${ }^{4}$. Linguisten könnten hier helfen ein deutliches Vokabular mitzuentwickeln, welches es vermeidet Begriffe zu entwickeln, die zu fern vom Alltag stehen. Auch könnten Linguisten vor allem in einem multilingualen Umfeld dazu beitragen, Begriffe so auszulegen, wie sie innerhalb der Verkehrauffassung der Parteien zu verstehen sind, also losgelöst von einem in die Vergangenheit gerichteten Gesetzesverständnis. Vor allem in gesetzlich noch nicht beschrittenen Rechtsgebieten kann die Auslegung durch Linguisten zu einem besseren Ergebnis führen, als bei einer Auslegung durch Juristen, die sich eventuell an eine analoge Sachverhaltsauslegung und Begriffsbestimmung gebunden fühlen, die nicht unbedingt der Verkehrsauffassung der Parteien entsprechen muss.

\subsection{Umgang mit dem Text: Hierarchie der Normen}

Linguisten und Juristen begegnen Texten auf unterschiedliche Weise. Ein Jurist koppelt Sachverhalte, wenn möglich, an Normen. Im Falle eines Begriffs, der aus sich selbst nicht deutlich oder präzise genug ist, wie zum Beispiel der Term ,unverzüglich“, würde ich als Juristin zunächst einmal schauen, ob der gleiche Begriff noch woanders im

4 Siehe z. B. für den Gebrauch von Begriffen, die dem Verbraucher nicht leicht zugänglich sind: KOM(2005) 483 endgültig, 2002/0222(COD), Geänderter Vorschlag für eine RICHTLINIE DES EUROPÄISCHEN PARLAMENTS UND DES RATES über Verbraucherkreditverträge und zur Änderung der Richtlinie 93/13/EWG. 
gleichen Gesetz gebraucht wird. Dann würde ich einen Kommentar anschauen, dann in einer Rechtssprechungssammlung auf Suche gehen, dann schauen, ob es gewohnheitsrechtliche Gebräuche gibt oder selbstregulatorische Maßnahmen des Sektors und dann würde ich mich in die einschlägige Rechtsliteratur einlesen. Allerdings kann diese Reihenfolge je nach Rechtssystem sehr unterschiedlich sein. Ein jedes System hat seine eigene Hierarchie der Rechtsquellen entwickelt. In den Niederlanden sind zum Beispiel Kommentare nicht so gebräuchlich wie in Deutschland. In England ist die Rechtsprechung wichtiger. In Schottland hat man ein gemischtes Rechtssystem, das der Rechtsprechung wieder einen anderen Platz zuweist als in England etc. Auch hier stellt sich dem Juristen nun wieder die Frage, ob linguistische Methoden alleine oder ergänzend eine Hilfestellung bei der Lösungsfindung bieten können, und ob eben diese Methoden entwickelt werden können durch ein linguistisches Monitoring?

\subsection{Linguistische Textprüfung}

Der Linguist wird die landesübliche Hierarchie der Normen nicht durchbrechen können. Die bohrende Frage ist nun, was kann ein Linguist zum Rechtsgewinn beitragen? Kann eine linguistische Textbewertung zu einem Rechtsgewinn und somit zu einer kohärenteren Rechtsauslegung und zu mehr Rechtssicherheit führen? Es sind mir keine Studien bekannt, die dies belegen. Hier besteht also noch Handlungsbedarf. Es wäre eine empirische Untersuchung notwendig, um zu belegen, was ein linguistisches Monitoring in der juristischen Praxis vermag.

Allerdings könnte der Linguist einen wertvollen Beitrag zur Entwicklung von sprachlichen Standards im Bereich von Rechtsentwicklungen auf der Ebene der Europäischen Union beitragen. Würden Linguisten und Juristen hier enger zusammenarbeiten, so ließe sich sicher mangelnde Kohärenz und Transparenz sowie eine Doppelnutzung von Begriffen in vielen Fällen vermeiden. Innerhalb Europas entwickelt sich losgelöst von der Landessprache eine Art „Kommissionssprache“ und auch ein „Kommissionsstil“. Häufig müssen die von der Europäischen Kommission abgefassten Texte später dann in den Mitgliedstaaten dem Bürger näher gebracht werden oder in das Landesrecht implementiert werden. Bei der Vereinigung von Kommissionssprache mit Landessprache könnten Linguisten bei der Schaffung von linguistischen Standards helfen. Linguisten sind daher vor allem im Be- 
reich von noch umzusetzenden EU Normen gefragt, die eben noch keinen Unterbau durch Rechtsprechung, Literatur und Kommentierung haben. Es ist zu vermuten, dass linguistische Methoden im Vorfeld der Umsetzung zu einem besseren Umsetzungsergebnis führen könnten.

\section{Gerechtfertigte Merkmale der Rechtssprache}

Juristen mussten in den vergangenen Jahrzehnten immer wieder auf neue Rechtsentwicklungen reagieren. Die internationale Zusammenarbeit der Staaten nahm zu und unzählige internationale Übereinkommen wurden unterzeichnet und zum Teil auch in das Landesrecht inkorporiert. Neben den internationalen Entwicklungen lief parallel die Integration in den Europäische Wirtschaftsraum. Auf diesen verschiedenen Ebene kamen die Juristen auch immer wieder in Kontakt mit Rechtstexten in anderen Sprachen. Regelmäßig mussten Konsense entwickelt werden und immer wieder Rechtstexte zu neuen Gebieten abgefasst werden. Man könnte nun denken, dass diese Entwicklungen auch zu einer Umorientierung in der Rechtssprache geführt haben, eben gerade durch zunehmende Abfassung von Rechtstexten in einer mehrsprachigen Kommission und durch die Übersetzung von Rechtstexten. Aber dennoch ist es festzustellen, dass die deutsche juristische Sprache weiterhin besondere Merkmale aufweist. Auf einige dieser Merkmale möchte ich hier kurz eingehen.

Immer wieder wird z. B. deutschen Juristen vorgeworfen, dass ihre Sätze zu lang und zu verschachtelt seien. In der Regel neigt der Jurist dazu, lange Sätze zu schreiben. Zumeist haben diese Sätze den Zweck, auf diese Weise Rückkoppelungen mit einzubauen. Dadurch soll im Fall der Interpretation auch die Ausnahme gleich im Satz vorkommen, um eine irreführende Interpretation von kurzen Sätzen zu vermeiden. Zerlegt man eine Regel in mehrere kürze Sätze, so ist schnell die Gefahr gegeben, dass zwar die Sätze deutlich sind, diese aber im Fall der Betrachtung Satz für Satz missdeutet werden könnten, da eben der Rückbezug auf die Ausnahme, die eben im Normalfall nur in einem verschachtelten Satz möglich ist, in einem in kürzere Sätze zerlegten Text nicht mehr gegeben ist. Dieses wäre für die Rechtssicherheit und den Rechtsgewinn ein wesentliches Problem, welches vermieden werden sollte. Verständlichkeitsoptimierung ist also nicht gleich Rechtsgewinn. 
Ein weiteres Merkmal der juristischen Sprache ist der häufig genutzte Nominalstil. Als Jurist neigt man leider zu diesem Stil. Der hat zumeist seinen Grund in einer gewissen Begriffsjurisprudenz. Viele Nomina sind Rechtsbegriffe, die so leichter zurückzufinden sind als in einem Verbstil. Als Beispiel sei hier der Satz: „A schließt mit B einen Kaufvertrag über ein Brot" gegeben. Der Jurist muss also gar nicht erst beginnen nachzudenken, um was für einen Vertrag es sich handelt. Der Text bietet die Lösung gleich an. Selbst ein ausländischer Jurist könnte so die Regel durch den Index im Gesetzbuch finden. Würde dieser Satz nun umgeschrieben in „A kauft von B ein Brot" so wäre das vielleicht kurz und schön, fordert aber eine unnötige Transferleistung vom Juristen, die durch einen Nominalstil vermieden werden könnte. Das gegebene Beispiel ist vielleicht ein wenig primitiv, aber es soll auch nur verdeutlichen, dass vor allem bei komplexeren Sachverhalten ein Nominalstil Rechtsdeutlichkeit schaffen kann und keinen unnötigen Raum für Interpretation entstehen lässt.

\section{Quantität versus Qualität}

Durch meine Arbeit in verschiedenen internationalen Gremien wird mir in der letzten Zeit immer bewusster, dass es ein großer Luxus ist, sich mit Fragen der Allgemeinverständlichkeit zu beschäftigen. Die europäische Rechtsharmonisierung überflutet den Juristen mit so einer Fülle von Texten und Informationen, dass die Probleme des Juristen in der Zukunft sicher vorerst einmal sein werden, überhaupt mit der Textmenge umgehen zu können und innerhalb Europas eine faire Sprachenregelung zu finden, die nicht einige Länder deutlich bevorzugt.

Ein gemeinsames Europa verlangt zum einen ständige Übersetzungsarbeiten und zum anderen eine kontinuierliche Implementierung von europäischen Rechtstexten in nationales Recht. ${ }^{5}$ Ohne Unterstützung von Außen erscheinen mir derzeit keine Kapazitäten mehr für eine

\footnotetext{
5 Auf europäischer Ebene erschien im Jahre 2002 zur Unterstützung der vielfältigen Rechtsumsetzungsprojekte das Dokument „Bessere Rechtsetzung“ (KOM(2002) 275 endgültig) und im selben Jahr die MITTEILUNG DER KOMMISSION Aktionsplan „Vereinfachung und Verbesserung des Regelungsumfelds“ (KOM(2002) 278 endgültig).
} 
sprachliche Verbesserung der Juristen-Laien Kommunikation von Seiten der Juristen frei zu sein. Eine langfristige Zusammenarbeit von Juristen und Linguisten wäre daher sehr zu begrüßen und ein linguistisches Monitoring kann dabei einen wertvollen Beitrag leisten. 


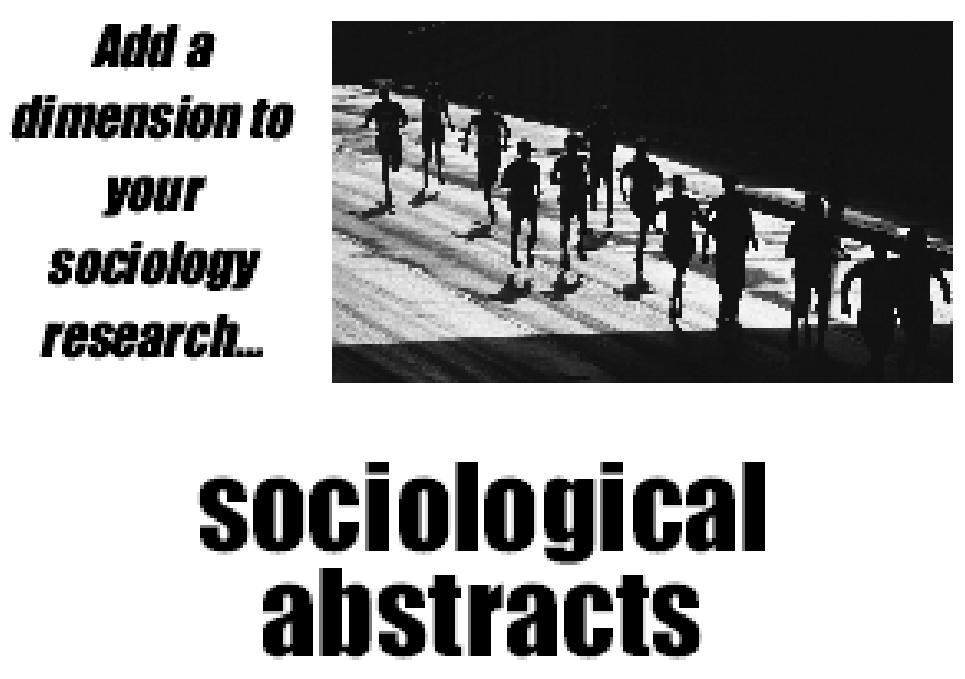

Comprehensive, cost-effective, timely coverage of current ideas in sociological research

Abstracts of articles, books, and conferenoe papers from nearly 2,000 journals published in 35 countries; citations of relevant dissertations as well as books and other media.

Wow featuring: - Gited references - Addilional alestracts Govesing 1963-1972

Available in print or electronically through CSA Illumina (www.csa.com).

Contact sales@csa.com for trial Internet access or a sample issue.

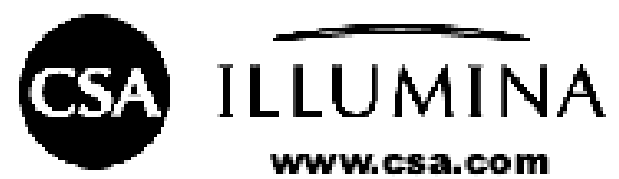

